\title{
The correlation between the severity of radiotherapy-induced glossitis and endothelial cell injury in local tissues in a rat model
}

\author{
Chun-yang Li ${ }^{1}$, Yun Hong ${ }^{2}$, Xiao-an Tao ${ }^{2}$, Juan Xia ${ }^{2}$, Bin Cheng ${ }^{2}$ \\ ${ }^{1}$ DDs, Ph.D, Department of Stomatology, The Fifth Affiliated Hospital of Sun Yat-sen University, Zhuhai,519000,China \\ ${ }^{2}$ DDs, Ph.D, Department of Oral Medicine, Guanghua School of Stomatology, Sun Yat-sen University, Guangzhou, 510060, \\ China
}

Correspondence:

Department of Oral Medicine,

The Guanghua School of Stomatology

The Affiliated Hospital of Stomatology,

Sun Yat-sen University,

Guangzhou, 510060, China

chengbin@mail.sysu.edu.cn

\begin{abstract}
Li CY, Hong Y, Tao XA, Xia J, Cheng B. The correlation between the severity of radiotherapy-induced glossitis and endothelial cell injury in local tissues in a rat model. Med Oral Patol Oral Cir Bucal. 2011 Sep 1;16 (6):e711-5.

http://www.medicinaoral.com/medoralfree01/v16i6/medoralv16i6p711.pdf
\end{abstract}

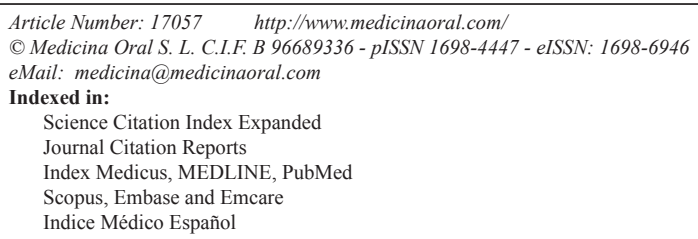

\begin{abstract}
Objectives: To explore the correlation between the severity of radiotherapy-induced glossitis (RTG) and endothelial cell injury in local tissues in a rat model.

Study Design: The RTG animal model was designed and used by our team. The Oral mucositis index(OMI) was documented daily. Immunohistochemistry (IHC) Staining of CD34 was utilized to identify endothelial cells in the RTG tissues. Apoptosis of endothelial cells in local lesions due to RTG was detected by the TUNEL assay. The dynamic relationship between the OMI and apoptotic endothelial cells was statistically analyzed by time.

Results and Conclusions: The injury and apoptosis of endothelial cells were observed 3 day post-irradiation. The vascular lumens of the post-irradiation tongue lesions were irregular; thrombosis formation in the center of the lumens, unsmooth lumen walls and vasodilated vessels were observed. Also, endothelial cells detached from the basal membrane and were found in the lumens. The percentages (\%) of apoptotic endothelial cells were $78.3 \pm 0.31$ (5 day); $89.3 \pm 0.83$ ( 8 day); $83.5 \pm 0.41$ (14 day); $69.3 \pm 0.57$ ( 21 day); and $47.3 \pm 0.59$ ( 28 day). The OMI was correlated with the percentage of apoptotic endothelial cells $(\mathrm{R}=0.67, \mathrm{P}=0.034)$. Summary, endothelial cell injury was correlated with the pathogenic condition of RTG.
\end{abstract}

Key words: Radiotherapy, oral mucositis, glossitis, endothelial cell, rat.

\section{Introduction}

Radiotherapy-induced glossitis (RTG) refers specifically to the injury of tongue tissues from radiotherapy for nasopharyngeal and other head and neck cancers. The clinical manifestations of RTG include hyperemia, erythema, ulceration, pseudomembrane formation and pain. Compared with more easily damaged sites, such as the buccal mucosa, oral floor or soft palate, the presentation of RTG is a more severe injury of the mucous membrane in the oral cavity (9). Therefore, an animal model of RTG was employed to produce obvious lesions in the tongue tissues (9-11). 
Through clinical observations and animal models, the basic initiation and progression of RTOM have been recognized. However, several evidence-based medical research studies have not identified effective prophylaxis or therapeutic measures, except palliative modalities $(12,13)$. At present, only one agent, Palifermin (recombinant keratinocyte growth factor-1), is approved for the treatment of cancer treatment-related oral mucositis (14). The pathogenesis of RTOM needs to be fully understood, so mechanism-based prophylaxis and therapeutic strategies can be developed $(5,15)$.

Research increasingly suggests that colony-forming cells in the basal layer of the mucosal epithelium (16) and other cells, such as fibroblasts and vascular endothelial cells in the submucosal tissue, are injured by irradiation $(9,17,18)$. The signaling of local tissue injury may communicate to the entire body through damaged blood vessels. Therefore, the aim of the present study was to explore the correlation between the severity of RTG and endothelial cell injury in local tissues in a rat model and to identify the role of vascular endothelial cell damage in the pathogenesis of RTG and its potential value as a pathway for developing prophylaxis or treatment.

\section{Materials and Methods \\ Ethics}

All procedures in this animal study were reviewed and approved by the Animal Ethics Committee at the Institute of Medical and Veterinary Sciences of Sun Yatsen University, Guangzhou, P.R. China. The work conducted on the animals and their care complied with the National Health and Medical Research Council Code of Practice of Care for Animals in Research and Training (1998).

\section{Animals and housing}

Thirty-six specific pathogen free (SPF), male SpragueDawley (SD) rats that were 10 to 12 weeks of age and weighed 250 to $300 \mathrm{~g}$ were purchased from the Northern Campus Animal Center of Sun Yat-sen University. The certification number of the experimental animal was SCXK (Guangdong) 2004-0011. The approved certification number was 2005A060.

Six rats were maintained in each polycarbonate cage and provided free access to standard rat food and filtered city tap water from standard Perspex ${ }^{\circledR}$ drinking bottles. A 12-hr-light/12-hr-darkness circadian rhythm was established using an automatic light program; the light phase lasted from 06:00 to 18:00 with lighting at 150-300 lux. The cages were kept in a room maintained at a temperature of $23 \pm 1{ }^{\circ} \mathrm{C}$ and a humidity of $55 \pm 5 \%$ under SPF conditions.

Irradiation Protocol and Experimental Design

All rats were allowed to adapt to breeding conditions for 2-3 days prior to $\mathrm{x}$-ray irradiation, so the maximum mit- igation of adverse irradiation responses was achieved. Rats were randomly divided into 6 groups of 6 with 1 group serving as the control and the other 5 groups as the experimental groups.

Prior to x-ray irradiation, each rat received an abdominal injection with $6 \mathrm{ml} / \mathrm{kg}$ of a $5 \%$ chloral hydrate solution. The 6 rats in the control group were shielded under a lead plate while the 30 rats in the 5 experimental groups were irradiated. To only irradiate the anterior dorsal tongue, we used a custom-built, cone-shaped, 2-mm thick lead device, which was designed and made in our laboratory. For each rat, the tongue was guided though an emargination on the lead device, and the anterior dorsal tongue was placed on the outer surface of the block and fixed with adhesive tape. An accessory lead plate with u-shaped emargination covered the tongue and limited the irradiation area to $10 \times 10 \mathrm{~mm}$. This two-layered lead plate ensured that the rest of the body was shielded from the ion-irradiation procedure. Irradiation settings of the deep x-ray machine (type F34-I; DongFang, Peking, P.R. China) were $210 \mathrm{kV}$ and $12 \mathrm{~mA}$ with a target distance of $40 \mathrm{~cm}$, an irradiation field of $10 \mathrm{~cm} \times 15 \mathrm{~cm}$, a $4 \mathrm{~mm}$ aluminum filter system. The $\mathrm{x}$-rays were delivered at $100.75 \mathrm{cGy} / \mathrm{min}$. The heads of the 6 rats were in the same irradiation field. The irradiation dosage was $30 \mathrm{~Gy}$ and delivered once at 9 a.m. Gross observation and times samples were obtained. All rats were monitored carefully twice daily throughout the experiment, increasing to four times daily if needed, with daily weight measurements, inspection of feces and assessment of behavioral characteristics at 8:00 a.m. and 3:00 p.m. Two cotton threads were used to open the oral cavity of the rat. The tongue was gently pushed out from the oral cavity with a cotton bud, and digital images were taken with a Fuji digital camera (Model E900) under a cold-lamp source. Clinical record sheets were filled out accordingly. An area on the dorsal surface of the tongue was calculated and evaluated with a previously described method (19) (Table 1) . According to the preliminary experiment, the experimental flowchart was determined (Fig. 1), and samples were obtained at each time.

Table 1. Oral mucositis scoring system adapted from the system proposed by Parkins (19).

\begin{tabular}{|c|l|}
\hline Score & \multicolumn{1}{|c|}{ Description } \\
\hline 0 & Normal \\
\hline 0.5 & Slightly pink \\
\hline 1.0 & Slightly red \\
\hline 2.0 & Severely red \\
\hline 3.0 & Focal desquamation \\
\hline 4.0 & $\begin{array}{l}\text { Exudate covering less than one-half of the } \\
\text { irradiated mucosa }\end{array}$ \\
\hline 5.0 & Virtually complete ulceration of the mucosa \\
\hline
\end{tabular}




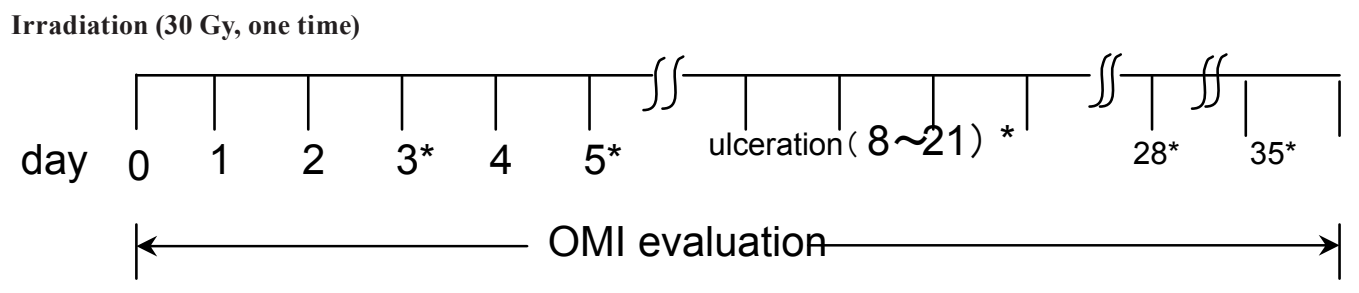

The times that samples were obtained: $5 \mathrm{~d}, 8 \mathrm{~d}, 14 \mathrm{~d}, 21 \mathrm{~d}$ and $28 \mathrm{~d}$ ( $\mathrm{n}=6$ for each group)

Fig. 1. Experimental flowchart.

At each time, 6 randomly selected rats in each group were exsanguinated via cardiac puncture and then killed via cervical dislocation. The tongue was cut at the tongue root. The tongue specimens were bathed in neutral buffered $10 \%$ concentrated formalin solution, embedded in paraffin, and excised for HE (Hematoxylin and Eosin Staining) staining and IHC (ImmunoHistoChemistry assay).

\section{Section preparation}

The tongue tissue samples were fixed with $4 \%$ neutral formalin, dehydrated and paraffin-embedded. For each block, three continuous serial 3- $\mu$ m thick sections were prepared. The first one was stained with HE. The second one was stained with IHC. The third one was analyzed with TUNEL.

\section{Histology (HE Staining)}

The first section of the three serial sections was stained with HE Staining (Mayer Kit, Germany) and observed under light microscopy.

\section{IHC Staining of CD34}

The second section of the three serial sections was stained with IHC staining with a CD34 mouse anti-rat monoclonal antibody (Santa Cruz, sc-7324) and observed under light microscopy. Briefly, antigen retrieval was accomplished by boiling a citric acid solution. A ready-to-use IHC rabbit anti-mouse LAB-SA kit (Tianjing Haoyang Biotechnique Co., MSP859143) was used for the assay. Arterial vessels provided by the kit company served as the positive control. The negative control was PBS, which replaced the first antibody added. Positive cells were stained a deep-yellow color.

Tunel Assay of Apoptotic Cells

The third section of the three serial sections was stained with the TUNEL assay (TUNEL Kit, Calbiochem). The sections were de-paraffinized with xylene twice for 5 minutes each, hydrated with $100 \%$ ethanol twice for 3 minutes each and 95\% ethanol for 1 minute and rinsed in distilled water. The sections were then pretreated by the proteinase $\mathrm{K}$ digestion method, incubated with $0.2 \%$ Triton X-100 in PBS-Tween for 30 minutes and Rinsed in PBS-Tween 20 twice for 2 minutes each. The sections were incubated in $3 \% \mathrm{H}_{2} \mathrm{O}_{2}$ in PBS for 10 minutes to block endogenous peroxidase activity and rinsed in PBS-Tween 20 three times for 2 minutes. The sections were incubated in a TdT reaction buffer for 10 minutes and then in a TdT Reaction Mixture for 2 hours at 37 ${ }^{\circ} \mathrm{C}$ in a humidified chamber. Next, they were rinsed in a stop wash buffer for 10 minutes to stop the reaction. After being rinsed in PBS-Tween 20 three times for 2 minutes, the sections were incubated with StreptavidinHRP in PBS for 20 minutes at room temperature and then rinsed in PBS-Tween 20 three times for 2 minutes. The sections were incubated with DAB for 1 minute, immediately rinsed in tap water, counterstained with Gill's hematoxylin for 30 seconds, rinsed in running tap water for 5 minutes and dehydrated in 95\% ethanol for 5 minutes and $100 \%$ ethanol twice for 3 minutes. Lastly, the sections were rinsed in xylene twice for 5 minutes, and a coverslip with a xylene-based mounting medium was used.

Counting apoptotic endothelial cells

The micro-blood vessels were determined within the three serial sections described above. The percentage of CD34-positive, apoptotic endothelial cells was counted with the TUNEL assay, apoptotic endothelial cells were counted under the high-power field $(\times 400)$. In 200 counted cells, the percentage of apoptotic cells was calculated. The above procedures were repeated three times for each group of serial sections.

Statistical analysis

All statistical data (OMI, percentage of apoptotic endothelial cells) were expressed as mean \pm SD. SPSS 16.0 statistics software was used to analyze the data. The one-way ANOVA statistical test was used to distinguish significance between groups. Results of the ANOVA were considered statistically significant when $\mathrm{P}<0.05$. In all the statistical tests conducted, data from the experimental groups were compared with data from the untreated control group. For the correlation analysis, Pearson's test was used. The significance level was $\mathrm{P}<0.05$.

\section{Results}

Submucosal blood vessel changes after irradiation Under the $\times 200$ visual field of microscopy, the vascular lumens in the control group were smooth on the internal surface, and endothelial cells were located on the wall of blood vessel wall. In the irradiated submucosal tissues, we observed rough, irregular vascular lumens, 
thrombosis, vasodilatation, increased permeability and detached endothelial cells (Fig. 2). Apoptotic endothelial cells were detected by the TUNEL assay (Fig. 3).

The correlation between the OMI and the percentage of apoptotic vascular endothelial cells The correlation coefficient between the percentage of apoptotic vascular endothelial cells and OMI at 5 day, 8 day, 14 day, 21 day and 28 day after irradiation was $0.67(\mathrm{P}=0.034)$ (Table 2$)$.
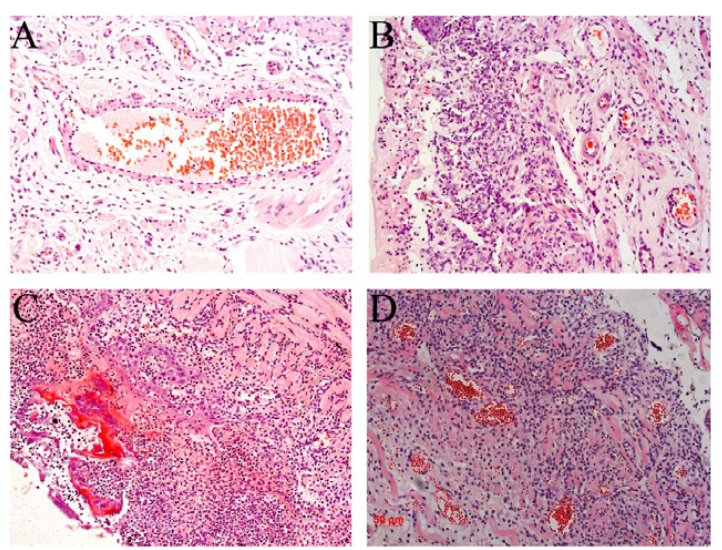

Fig. 2. Changes in the submucosal blood vessels of the tongue after irradiation. A: 3 day post-irradiation; B: 8 day post-irradiation; C: 18 day post-irradiation; D: 21 day post-irradiation.

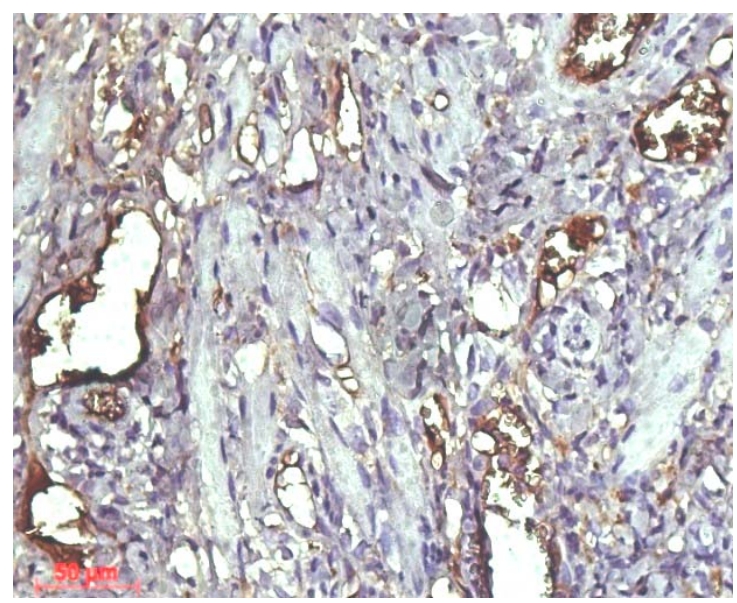

Fig. 3. Cellular apoptosis detected by the TUNEL assay. The brown-staining cells showed the apoptotic vascular endothelial cells at 8 day post-irradiation.

Table 2. The correlation assay between the percentage of apoptotic vascular endothelial cells and the OMI of rat tongue after irradiation.

\begin{tabular}{|c|c|c|}
\hline Groups & $\begin{array}{c}\text { Percentage of apoptotic } \\
\text { endothelial cells }\end{array}$ & OMI \\
\hline 5 day post-irradiation $(\mathrm{n}=6)$ & $78.3 \pm 0.31$ & $1.83 \pm 0.41$ \\
\hline 8 day post-irradiation $(\mathrm{n}=6)$ & $89.3 \pm 0.83$ & $3.37 \pm 0.82$ \\
\hline 14 day post-irradiation $(\mathrm{n}=6)$ & $83.5 \pm 0.41$ & $4.83 \pm 0.41$ \\
\hline 21 day post-irradiation $(\mathrm{n}=6)$ & $69.3 \pm 0.57$ & $4.50 \pm 0.55$ \\
\hline 35 day post-irradiation $(\mathrm{n}=6)$ & $47.3 \pm 0.59$ & $2.50 \pm 0.55$ \\
\hline
\end{tabular}

\section{Discussion}

Previous research have found that an important area of the mucosal tissue that is vulnerable to radiation injury is the submucosal micro-blood, especially endothelial cells $(9,15,17)$. This might contribute to the pathogenesis of radiotherapy-induced mucosal ulceration. In the present study, endothelial cell injury was observed prior to injury of the mucosal epithelium, which was indicated by the appearance of mucosal ulceration. Endothelial cell injury occurred 3 day after irradiation whereas injury of the mucosal epithelium occurred 5 day post-irradiation. While the mucosal epithelium was intact, the integrity of the submucosal blood vessels was destroyed, and the endothelial cells were detached from the wall of the micro-blood vessels.

The lumens of the blood vessels were irregular and contained thrombi. The following two reasons may explain the phenomenon. First, endothelial cell injury causes mucosal epithelial cell death. The supply of oxygen and other nutrients to the epithelial cells was affected by endothelial cell damage. Micro-vascular permeability increased, leading to edema, hyperemia in the submucosa and the erythematous appearance. Wearing et al. (20) established a mathematic model of vascular endothelial cells and mucosal epithelial cells and showed that endothelial cells secreting keratinocyte growth factors triggered the growth and differentiation of epithelial cells. When the endothelial cells were damaged by irradiation, the normal signaling that promotes growth and differentiation of the epithelial cells was lost, possibly resulting in the atrophy and thinning of the epithelium. Secondly, endothelial cells are more sensitive to irradiation than epithelial cells, so endothelial cells are damaged first.

There are different arguments regarding the causes of the radiation gastrointestinal (GI) syndrome: the endothelial cells lining the blood vessels of the villi and the crypts of the jejunum or the intestinal epithelial cells, the colony-forming cells, in the crypts. The issues debated are fundamentally important because they underlie the basic mechanism by which normal tissues are damaged by irradiation. In the 1970s, the pioneering studies of Withers et al. (21) proposed that the radiation GI syndrome was caused by death of the cells of the crypts of Lieberkühn, sterilization by radiation of the stem cells of a normal tissue. This dogma was challenged by a Science paper in 2001 written by Paris et al. (17), who suggested that that the endothelium within the intestinal mucosa was the actual target of radiation damage, in turn, causing stem cell dysfunction (17). Recently, Schuller reported no significant endothelial cell apoptosis in the radiation-induced GI syndrome (18). López-Castaño et al. (22) and Rotolo et al. (23)also observed that the absence of Bax and Bak, which abrogate endothelial cell apoptosis, protected the crypts from ra- 
diation radiation damage, but only by a dose modification factor (DMF) of roughly 1.15. To our knowledge, as in our study, endothelial cell injury possibly plays a role in early, but not late, tissue injury.

As mentioned previously, the tongue tissue is a site for severe oral mucositis. The OMI scores the severity of the oral mucosal injury. Based on the correlation assay, the correlation coefficient between the percentage of apoptotic vascular endothelial cells and the OMI of rat tongues after irradiation was $0.67(\mathrm{P}=0.034)$. Five to eight days post-irradiation, damage from the RTG (based on the OMI) increased as endothelial cell injury increased. At 9 day to 21 day after irradiation, the speed of endothelial cell injury slowed down, but radiation damages of the epithelium reached their peak. The above results suggest that early tissue injury occurred at the submucosa, and endothelial cell injury was involved in the process.

\section{References with links to Crossref - DOI}

\section{References}

1. Vissink A, Jansma J, Spijkervet FK, Burlage FR, Coppes RP. Oral sequelae of head and neck radiotherapy. Crit Rev Oral Biol Med. 2003;14:199-212.

2. Sciubba JJ, Goldenberg D. Oral complications of radiotherapy. Lancet Oncol. 2006;7:175-83.

3. Trotti A, Bellm LA, Epstein JB, Frame D, Fuchs HJ, Gwede CK, et al. Mucositis incidence, severity and associated outcomes in patients with head and neck cancer receiving radiotherapy with or without chemotherapy: a systematic literature review. Radiother Oncol. 2003;66:253-62.

4. Rubenstein EB, Peterson DE, Schubert M, Keefe D, McGuire D, Epstein J, et al. Clinical practice guidelines for the prevention and treatment of cancer therapy-induced oral and gastrointestinal mucositis. Cancer. 2004;100:2026-46.

5. Scully C, Sonis S, Diz PD. Oral mucositis. Oral Dis. 2006;12:22941.

6. Vera-Llonch M, Oster G, Hagiwara M, Sonis S. Oral mucositis in patients undergoing radiation treatment for head and neck carcinoma. Cancer. 2006;106:329-36.

7. Keefe DM, Schubert MM, Elting LS, Sonis ST, Epstein JB, RaberDurlacher JE, et al. Updated clinical practice guidelines for the prevention and treatment of mucositis. Cancer. 2007;109:820-31.

8. Rosenthal DI, Trotti A. Strategies for managing radiation-induced mucositis in head and neck cancer. Semin Radiat Oncol. 2009;19:2934.

9. Zhao J, Kim KA, De Vera J, Palencia S, Wagle M, Abo A. R-Spondin1 protects mice from chemotherapy or radiation-induced oral mucositis through the canonical Wnt/beta-catenin pathway. Proc Natl Acad Sci U S A. 2009;106:2331-6.

10. Dörr W, Kummermehr J. Accelerated repopulation of mouse tongue epithelium during fractionated irradiations or following single doses. Radiother Oncol. 1990;17:249-59.

11. Dörr W, Emmendörfer H, Haide E, Kummermehr J. Proliferation equivalent of 'accelerated repopulation' in mouse oral mucosa. Int $\mathrm{J}$ Radiat Biol. 1994;66:157-67.

12. Stokman MA, Spijkervet FK, Boezen HM, Schouten JP, Roodenburg JL, de Vries EG. Preventive intervention possibilities in radiotherapy- and chemotherapy-induced oral mucositis: results of metaanalyses. J Dent Res. 2006;85:690-700.

13. Worthington HV, Clarkson JE, Eden OB. Interventions for preventing oral mucositis for patients with cancer receiving treatment. Cochrane Database Syst Rev. 2007;4:CD000978.

14. Spielberger R, Stiff P, Bensinger W, Gentile T, Weisdorf D, Kewalramani $\mathrm{T}$, et al. Palifermin for oral mucositis after intensive ther- apy for hematologic cancers. N Engl J Med. 2004;351:2590-8. 15. Sonis ST. The pathobiology of mucositis. Nat Rev Cancer. 2004;4:277-84.

16. Bloomer WD, Hellman S. Normal tissue responses to radiation therapy. N Engl J Med. 1975;293:80-3.

17. Paris F, Fuks Z, Kang A, Capodieci P, Juan G, Ehleiter D, et al. Endothelial apoptosis as the primary lesion initiating intestinal radiation damage in mice. Science. 2001;293:293-7.

18. Schuller BW, Rogers AB, Cormier KS, Riley KJ, Binns PJ, Julius $\mathrm{R}$, et al. No significant endothelial apoptosis in the radiationinduced gastrointestinal syndrome. Int J Radiat Oncol Biol Phys. 2007;68:205-10

19. Parkins CS, Fowler JF, Yu S. A murine model of lip epidermal/ mucosal reactions to X-irradiation. Radiother Oncol. 1983;1:159-65. 20. Wearing HJ, Sherratt JA. Keratinocyte growth factor signalling: a mathematical model of dermal-epidermal interaction in epidermal wound healing. Math Biosci. 2000;165:41-62.

21. Withers HR, Elkind MM. Microcolony survival assay for cells of mouse intestinal mucosa exposed to radiation. Int J Radiat Biol Relat Stud Phys Chem Med. 1970;17:261-7.

22. López-Castaño F, Oñate-Sánchez RE, Roldán-Chicano R, Cabrerizo-Merino MC. Measurement of secondary mucositis to oncohematologic treatment by means of different scale. Review. Med Oral Patol Oral Cir Bucal. 2005;10:412-21.

23. Rotolo JA, Maj JG, Feldman R, Ren D, Haimovitz-Friedman A, Cordon-Cardo C, et al. Bax and Bak do not exhibit functional redundancy in mediating radiation-induced endothelial apoptosis in the intestinal mucosa. Int J Radiat Oncol Biol Phys. 2008;70:804-15. 